\title{
MIRROS: a randomized, placebo-controlled, Phase III trial of cytarabine \pm idasanutlin in relapsed or refractory acute myeloid leukemia
}

\author{
Pau Montesinos*,1,2, Benjamin M Beckermann ${ }^{3}$, Olivier Catalani ${ }^{3}$, Jordi Esteve ${ }^{4}$, Katia \\ Gamel $^{3}$, Marina Y Konopleva5 ${ }^{5}$ Giovanni Martinelli ${ }^{6}$, Annabelle Monnet ${ }^{3}$, Cristina \\ Papayannidis $^{7}$, Aaron Park ${ }^{8}$, Christian Récher ${ }^{9}$, Rebeca Rodríguez-Veiga ${ }^{1}$, Christoph \\ Röllig ${ }^{10}$, Norbert Vey ${ }^{11}$, Andrew H Wei ${ }^{12}$, Sung-Soo Yoon ${ }^{13}$ \& Pierre Fenaux ${ }^{14}$ \\ ${ }^{1}$ Departamento de Hematologia, Hospital Universitari i Politècnic La Fe, València, Spain \\ ${ }^{2}$ CIBERONC, Instituto Carlos III, Madrid, Spain \\ ${ }^{3}$ F. Hoffmann-La Roche Ltd, Basel, Switzerland \\ ${ }^{4}$ Hospital Clinic de Barcelona, IDIBAPS, Barcelona, Spain \\ ${ }^{5}$ Department of Leukemia, Division of Cancer Medicine, The University of Texas MD Anderson Cancer Center, Houston, TX, USA \\ ${ }^{6}$ Department of Hematology and Sciences Oncology, Istituto Scientifico Romagnolo per lo Studio e la Cura dei Tumori (IRST) \\ IRCCS, Meldola, Italy \\ ${ }^{7}$ Department of Experimental, Diagnostic and Specialty Medicine Institute of Hematology \& Medical Oncology L \& A Seràgnoli, \\ Bologna, Italy \\ ${ }^{8}$ Hoffmann-La Roche Ltd, Mississauga, ON, Canada \\ ${ }^{9}$ Serviced'Hématologie, Institut Universitaire du Cancer Toulouse - Oncopole, Toulouse, France \\ ${ }^{10}$ Department of Internal Medicine, Universitätsklinikum Carl Gustav Carus, Dresden, Germany \\ ${ }^{11}$ Hematology Department, Aix-Marseille University, Institut Paoli-Calmettes, Marseille, France \\ ${ }^{12}$ Department of Haematology, The Alfred Hospital \& Monash University, Melbourne, VIC, Australia \\ ${ }^{13}$ Division of Hematology/Medical Oncology, Department of Internal Medicine, Seoul National University Hospital, Seoul, South \\ Korea \\ ${ }^{14}$ Service d'Hématologie Séniors Hôpital Saint-Louis, Assistance Publique - Hôpitaux de Paris, Université de Paris, Paris, France \\ *Author for correspondence: Tel.: +34 96124 4925; Fax: +34 96 124 6201; montesinos_pau@gva.es
}

Patients with refractory or relapsed acute myeloid leukemia (R/R AML) have a poor prognosis, with a high unmet medical need. Idasanutlin is a small-molecule inhibitor of MDM2, a negative regulator of tumor suppressor p53. By preventing the p53-MDM2 interaction, idasanutlin allows for p53 activation, particularly in patients with TP53 wild-type (WT) status. MIRROS (NCT02545283) is a randomized Phase III trial evaluating idasanutlin + cytarabine versus placebo + cytarabine in $\mathrm{R} / \mathrm{R}$ AML. The primary end point is overall survival in the TP53-WT population. Secondary end points include complete remission rate (cycle 1), overall remission rate (cycle 1) and event-free survival in the TP53-WT population. MIRROS has an innovative design that integrates a stringent interim analysis for futility; continuation criteria were met in mid-2017 and accrual is ongoing. Trial registration number: NCT02545283

First draft submitted: 17 January 2020; Accepted for publication: 12 February 2020; Published online: 13 March 2020

Keywords: acute myeloid leukemia $\bullet$ AML $\bullet$ cytarabine $\bullet$ idasanutlin $\bullet$ MDM2 $\bullet$ MIRROS • p53 $\bullet$ refractory $\bullet$ relapsed - trial in progress

Acute myeloid leukemia (AML) is a malignant and genetically heterogeneous hematologic disease in which uncontrolled clonal expansion of myeloid blast cells occurs in the bone marrow, blood and other tissues $[1,2]$. The age-adjusted incidence rate of AML in the USA in 2016 was 4.3 per 100,000 person-years, with similar rates in the UK, Australia, Canada and other European countries (e.g., Sweden, Denmark) [3]. The 5-year survival rates for younger (age 18-60 years) and elderly (age >60 years) patients with AML are 40 and 10\%, respectively [2]. With 


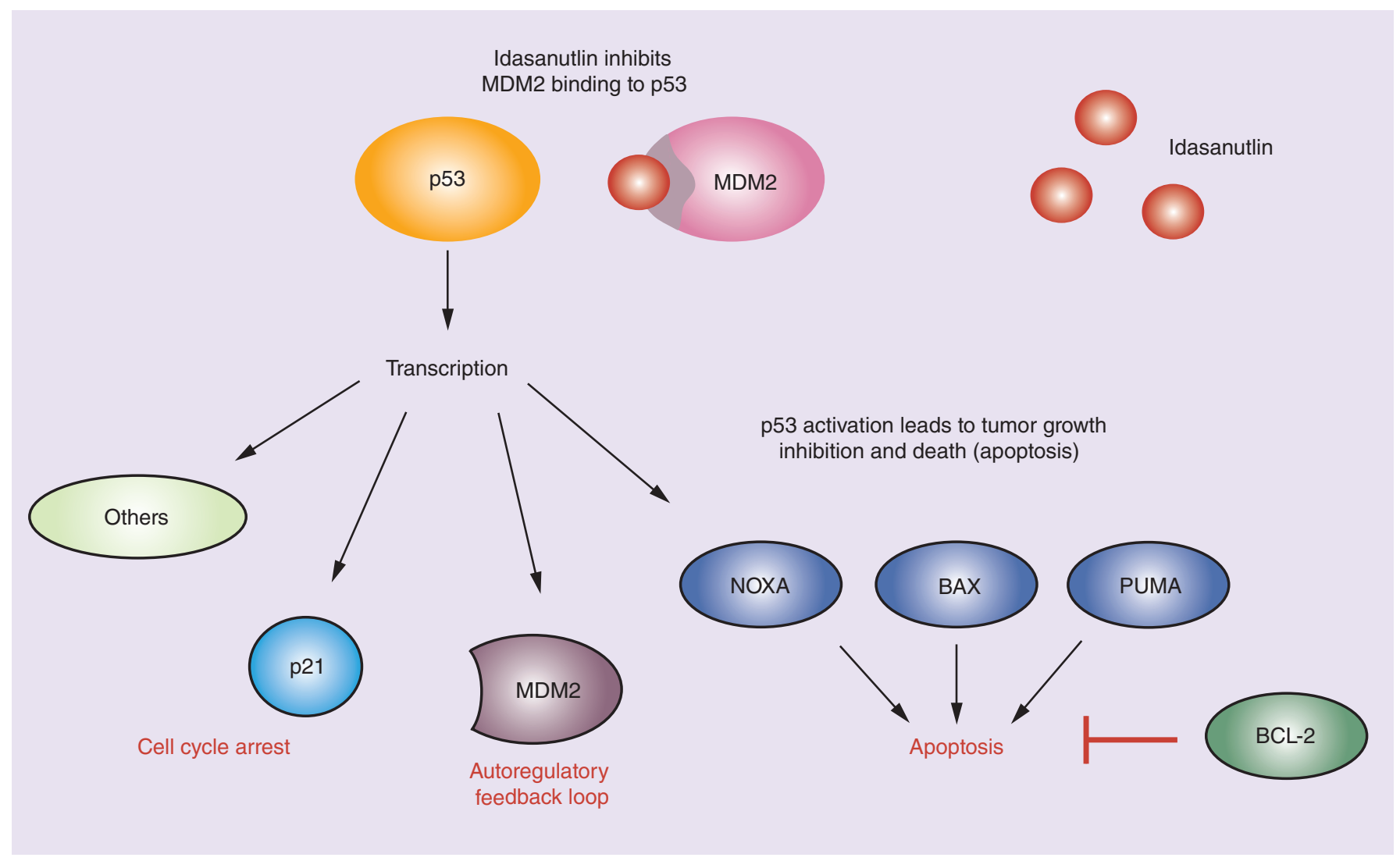

Figure 1. Idasanutlin: p53 activation via MDM2 inhibition.

intensive chemotherapy, approximately $60-80 \%$ of patients achieve complete remission; however, nearly a quarter will have primary refractory disease and more than half will relapse, typically within 3 years from initial response [46]. For patients with refractory or relapsed (R/R) AML, there is no standard treatment regimen. Recommended therapies in R/R AML include intensive chemotherapy followed by allogeneic hematopoietic stem cell transplantation (HSCT) in patients fit enough to tolerate aggressive treatment, hypomethylating agents or best supportive care in the unfit patient population [7,8]. Targeted therapies for select patients with certain genetic profiles such as FLT3-, IDH1- or IDH2-mutated AML have also become available [8]. Despite these novel therapeutic approaches to $\mathrm{R} / \mathrm{R}$ AML, the prognosis in this population is poor [4] and a high unmet medical need remains.

TP53 is a tumor suppressor gene, which is frequently mutated or deleted in human cancers, leading to a dysfunctional p53 tumor suppressor protein and facilitating uncontrolled cell proliferation [9]. In addition, p53 can be inactivated through protein degradation, which is triggered by binding to the E3 ligase MDM2, the main negative regulator of $\mathrm{p} 53$ [10-12]. Indeed, overexpression of $M D M 2$ has been observed in human tumors [13]. Therefore, in cancers with wild-type TP53 (TP53-WT), which includes a large proportion of patients with AML $(\approx 80 \%)_{[14-16]}$, targeting MDM2 may be a promising therapeutic strategy to stabilize p53 levels and activate p53 tumor suppressor function and downstream apoptotic pathways (Figure 1).

\section{Idasanutlin}

Idasanutlin is a novel, potent and selective small-molecule MDM2 antagonist with a pyrrolidine structure [17,18]. It has an identical cellular mechanism to other Nutlin family molecules; however, enhanced potency, selectivity and bioavailability have been observed with the novel chemistry of idasanutlin [17]. Preclinical evaluations showed induction of cell cycle arrest and/or apoptosis with idasanutlin in tumor cell lines [18]. In addition, antitumor activity was observed with both idasanutlin monotherapy and combination therapy with cytarabine in xenograft models [18]. 


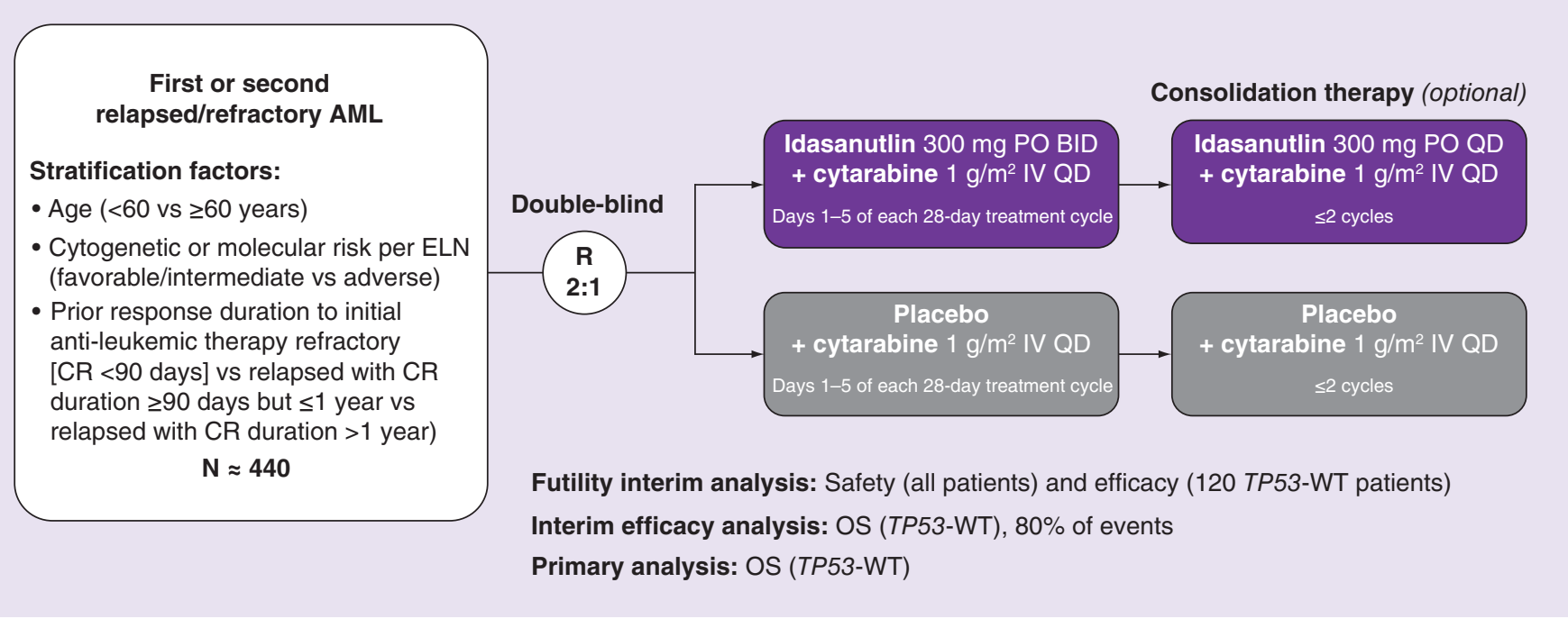

Figure 2. MIRROS study design.

AML: Acute myeloid leukemia; CR: Complete remission; ELN: European LeukemiaNet; HSCT: Hematopoietic stem cell transplant; IV: Intravenously; OS: Overall survival; PO: Orally; QD: Once daily; R: Randomized; WT: Wild-type.

In a multicenter, open-label, Phase I/Ib trial in patients with AML (NCT01773408), idasanutlin (400-1600 mg daily for 5 days) demonstrated tolerable safety and encouraging clinical activity alone and in combination with cytarabine ( $1 \mathrm{~g} / \mathrm{m}^{2}$ iv. for 6 days) [16,19]. This study enrolled patients regardless of TP53 mutation status, as some mutations in TP53 allow p53 functionality to be retained [20,21]. Adverse events (AEs) reported across treatment cohorts included gastrointestinal events, hematologic events or events related to infections or infestations [16,19]. Grade $\geq 3$ AEs included hypokalemia, diarrhea, nausea and febrile aplasia or neutropenia [16,19]. No unexpected toxicities were reported with the combination regimen compared with other relapse regimens containing cytarabine [16,22,23]. Overall response rate (ORR; complete remission $[\mathrm{CR}]+\mathrm{CR}$ with incomplete platelet recovery [CRp] + CR with incomplete blood count recovery [CRi] + morphologic leukemia free state) in the monotherapy cohorts was $15 \%$ $(\mathrm{n}=7 / 46)$ [19]. In the combination cohorts, ORR was 33\% $(\mathrm{n}=25 / 75)$ [16]. The majority of responses were achieved after one cycle of treatment [16,19]. In the combination cohorts, median duration of response for patients with CR/CRp/CRi followed up to 1 year was $>8$ months [16].

Preliminary findings from this study led to the continuing evaluation of idasanutlin in combination with cytarabine in R/R AML in the Phase III MDM2 Inhibitor in R/R AML for Overall Survival (MIRROS) trial (NCT02545283).

\section{MIRROS trial}

\section{Study design}

MIRROS is a multicenter, randomized, double-blind, Phase III trial evaluating idasanutlin in combination with cytarabine versus placebo in combination with cytarabine in patients with R/R AML (Figure 2). Approximately 440 patients will be enrolled across 80 centers and 18 countries in the Americas, Europe and Asia-Pacific region. Patients are being randomized 2:1 to receive idasanutlin $300 \mathrm{mg}$ orally twice daily and cytarabine $1 \mathrm{~g} / \mathrm{m}^{2} \mathrm{iv}$. once daily on days $1-5$ of each 28-day treatment cycle versus placebo and cytarabine $1 \mathrm{~g} / \mathrm{m}^{2}$ iv. once daily on days 1-5 of each 28-day treatment cycle; this dosing regimen is based on results of the Phase Ib study that investigated tolerability and clinical activity [16,19]. The initial induction cycle is 28 days and a maximum of two additional cycles of consolidation therapy with idasanutlin/placebo $300 \mathrm{mg}$ orally once daily and cytarabine iv. may be administered to patients who respond to treatment. Antibiotic, antifungal, antidiarrheal and anti-emetic prophylaxis during treatment cycles is required. Stratification factors are age ( $<60 \mathrm{vs} \geq 60$ years), cytogenetic/molecular risk per 2010 European LeukemiaNet recommendations [24] (favorable/intermediate vs adverse), prior HSCT (yes or no) and prior response duration to initial antileukemic therapy (refractory [CR $<90$ days] vs relapsed with CR duration $\geq 90$ days but $\leq 1$ year vs relapsed with CR duration $>1$ year). For eligibility and response assessments, AML disease is confirmed locally based on bone marrow aspirate or biopsy review. Following randomization screening bone 
Table 1. Futility interim analysis criteria.

\begin{tabular}{|c|c|c|}
\hline Results & GO & STOP \\
\hline Efficacy (futility; 120 TP53-WT patients) & $\begin{array}{l}\text { - } \mathrm{CR}^{\dagger} \text { odds ratio } \geq 2.5 \text { or } \\
\text { - } \mathrm{CR}^{\dagger} \text { odds ratio } \geq 2.0 \text { and } \mathrm{EFS} \mathrm{HR} \leq 1\end{array}$ & $\begin{array}{l}-\mathrm{CR}^{\dagger} \text { odds ratio }<2.0 \text { or } \\
-\mathrm{CR}^{\dagger} \text { odds ratio } \geq 2.0 \text { and }<2.5 \text { and EFS HR }>1 \\
\text { Probability (stop if drug does not work) }=90 \%\end{array}$ \\
\hline Safety (all patients) & $\begin{array}{l}- \text { Gl toxicity } \ddagger \text { : grade } 3 \leq 40 \% \text {; grade } 4 \leq 15 \% \text { and } \\
-<10 \% \text { difference in early death rate }(\leq \text { day } 30) \text { in } \\
\text { treatment vs control ( } \leq 20 \% \text { early death rate) }\end{array}$ & $\begin{array}{l}- \text { GI toxicity }{ }^{\ddagger} \text { : grade } 3>40 \% \text {; grade } 4>15 \% \text { or } \\
\bullet \geq 10 \% \text { difference in early death rate ( } \leq \text { day } 30 \text { ) in } \\
\text { treatment vs control ( }>20 \% \text { early death rate) }\end{array}$ \\
\hline \multicolumn{3}{|c|}{$\begin{array}{l}\dagger \text { Defined as CR or CR with incomplete platelet recovery with a duration of } \geq 28 \text { days. } \\
\text { ‡Defined as nausea, vomiting and diarrhea. }\end{array}$} \\
\hline
\end{tabular}

marrow samples are tested centrally using a next-generation sequencing diagnostic platform to identify populations for study objectives (e.g., TP53, IDH1 and IDH2 mutational status). Post-treatment bone marrow specimens are centrally evaluated for minimal residual disease using flow cytometry and polymerase chain reaction technology.

An interim efficacy analysis on overall survival (OS) is planned to be conducted by the independent data monitoring committee (iDMC) after $80 \%$ of death events have been observed in the TP53-WT population to allow for early testing of OS and data unblinding, if relevant efficacy is achieved.

MIRROS is unique in that it integrates Phase II safety and efficacy criteria into a Phase III study via a blinded interim analysis for futility. This study design feature mitigates the risk of moving directly from a Phase Ib to a Phase III trial $[25,26]$.

During the design phase, an important challenge of the interim futility analysis was assessing the magnitude of efficacy benefit because infection-related death in patients with AML and transplant-related mortality increase the risk of death without clear causality to study treatment after randomization. Thus, if the observation period for OS was not long enough, the treatment-effect comparison between the arms would be weighted toward early death events. Therefore, MIRROS was designed with durable CR (defined as CR or CRp with a duration of $\geq 28$ days) as an intermediate efficacy end point in the interim futility analysis. Though not fully recognized as a surrogate end point, CR as an intermediate end point is associated with the primary end point of OS [27].

The interim futility analysis utilized a high bar on the odds ratio for durable CR and event-free survival (EFS), supporting the association between CR and OS to increase the quality of the futility assessment and included gating criteria to evaluate safety (Table 1). The futility analysis was performed by an iDMC after 120 patients with TP53WT were enrolled, received $\geq 80 \%$ of the first treatment cycle and had a confirmatory response assessment available or who had withdrawn before response/confirmation; patients with TP53-WT were assessed for efficacy and all patients were assessed for safety. The prespecified number of 120 patients was chosen because it was considered sufficient for an interim decision and mirrored the sample size of a randomized Phase II trial [26]. Both the trial sponsors and investigators were blinded to the iDMC-reviewed data, thus maintaining trial integrity and avoiding the introduction of bias.

Per the nonbinding efficacy futility criteria, the trial would continue if the confirmed CR odds ratio was $\geq 2.5$ or $\geq 2.0$ with an EFS hazard ratio of $\leq 1$ (Table 1 ). With regard to safety, the trial would continue if the proportion of gastrointestinal toxicity (nausea, vomiting or diarrhea) in the idasanutlin plus cytarabine arm was $\leq 40 \%$ of grade 3 or $\leq 15 \%$ of grade 4 events, there was $<10 \%$ difference in the proportions of early deaths ( $\leq 30$ days from randomization) between treatment arms or $\leq 20 \%$ of early deaths occurred in the idasanutlin plus cytarabine arm (Table 1). A relatively high false-negative error probability was considered in the interim boundary selection; thus, setting a high bar for futility and providing increased confidence in the experimental arm should the trial pass the thresholds [26].

\section{Comparator arm}

Cytarabine is an analog of cytosine, which may impede DNA synthesis, cause cellular damage and may thus enhance the antitumor activity of MDM2 inhibitors [24,28]. In the R/R setting, cytarabine is effective and generally used in combinations at doses ranging from $1-2 \mathrm{~g} / \mathrm{m}^{2}[6,8,29]$. In MIRROS, cytarabine $1 \mathrm{~g} / \mathrm{m}^{2}$ once daily (over 1-3 h) was chosen in combination with idasanutlin based on findings from a Phase Ib study that evaluated the combination of cytarabine at low $\left(20 \mathrm{mg} / \mathrm{m}^{2}\right.$ subcutaneous $)$ and intermediate $\left(1 \mathrm{~g} / \mathrm{m}^{2}\right.$ once daily $\times$ days $\left.1-6\right)$ doses with MDM2-antagonist, RG7112 [30]. Doses of cytarabine greater than $1 \mathrm{~g} / \mathrm{m}^{2}$ daily were considered too toxic and unlikely to provide substantial benefit in the expected patient population [31,32]. 
Table 2. Key eligibility criteria.

Inclusion criteria

\section{- Age $\geq 18$ years}

- Confirmed primary or secondary refractory ${ }^{\dagger}$ or relapsed AML per WHO classification, except APL

- $\leq 2$ prior induction regimens (excluding prior HSCT) in first-line treatment, including cytarabine with an anthracycline (or anthracenedione)

- ECOG PS 0-2

\section{Exclusion criteria}

- First relapsed patients aged $<60$ years with a first $C R$ duration of $>1$ year

- Prior documented AHD, including myelodysplastic syndromes, myeloproliferative disease (i.e., CMML, PV, primary MF, ET) and aplastic anemia

- AML secondary to any prior chemotherapy unrelated to leukemia

- Refractory to or has relapsed within 90 days of receiving a regimen containing a cumulative dose of cytarabine $\geq 18 \mathrm{~g} / \mathrm{m}^{2}$

- Allogeneic $\mathrm{HSCT} \leq 90$ days prior to randomization

- Prior treatment with an MDM2 antagonist

- Infection considered by the investigator to be clinically uncontrolled (e.g., patients who are or should be on antimicrobial agents for treatment of active infection)

†Primary refractory: lack of response after 1-2 induction cycles in front line; secondary refractory: lack of response after one line of treatment for relapsed/refractory AML.

AHD: Antecedent hematological disorder; AML: Acute myeloid leukemia; APL: Acute promyelocytic leukemia; CMML: Chronic myelomonocytic leukemia; CR: Complete remission; ECOG PS: Eastern Cooperative Oncology Group performance status; ET: Essential thrombocythemia; HSCT: Hematopoietic stem cell transplantation; MF: Myelofibrosis; PV: Polycythemia vera.

There is no global standard treatment regimen for R/R AML and prior studies did not show significant difference in OS between chemotherapy regimens including a $1 \mathrm{~g} / \mathrm{m}^{2}$ comparator [33]. Choice of a placebo in combination with the cytarabine comparator was therefore considered ethical and acceptable for the blinded study design and facilitated establishing the toxicity profile of the experimental arm.

To have fewer patients assigned to placebo, the randomization ratio is 2:1 in favor of the experimental arm. Eligibility criteria were also introduced to exclude patients who are refractory to or who have relapsed within 90 days of receiving a cumulative dose of $>18 \mathrm{~g} / \mathrm{m}^{2}$ cytarabine, as they were unlikely to benefit from treatment with the comparator. Young patients with a first CR of $>1$ year as higher intensity chemotherapy rather than single-agent cytarabine dose was considered a more appropriate treatment option for those patients.

\section{Eligibility criteria}

Key inclusion and exclusion criteria are listed in Table 2. Overall, eligible patients are aged $\geq 18$ years and have confirmed first or second R/R AML per WHO classification [34]. An Eastern Cooperative Oncology Group performance status $\leq 2$, acceptable hepatic/renal function and $\leq 2$ prior induction regimens (excluding HSCT) for first-line therapy, including cytarabine with an anthracycline or anthracenedione, are also required. The exclusion of first-relapsed patients aged $<60$ years with a first CR duration of $>1$ year from enrollment omits patients in whom standard practice would be to use intensive chemotherapy. Patients treated with $>2$ prior induction regimens as first-line therapy or who had documented refractory disease or relapse(s) within 90 days of receiving a regimen including a cumulative dose of cytarabine $\geq 18 \mathrm{~g} / \mathrm{m}^{2}$ are excluded to limit the study population to patients with the potential to respond to the $1 \mathrm{~g} / \mathrm{m}^{2}$ intermediate once daily iv. (over 1-3 h) dose of cytarabine. Patients with secondary AML were also excluded from this study. As not every TP53 mutation negatively affects p53 functionality [35], patients are being enrolled regardless of TP53 mutation status.

\section{Study end points \& outcome measures}

The primary end point of this study is OS in the TP53-WT population. Secondary efficacy end points in the TP53-WT population include CR rates per investigator during the induction cycle, EFS, overall remission rates (CR, CRp and CRi) during the induction cycle, duration of CR, the proportions of allogeneic HSCT following CR and $O S$ and CR in the subpopulation with clinically actionable mutations (i.e., FLT3, IDH1 and IDH2). Additional study end points are safety, pharmacokinetics (PK) and patient-reported outcomes (PROs) using the European Organisation for the Research and Treatment of Cancer Quality of Life Questionnaire Core 30 (EORTC QLQ-C30) and EuroQol 5 Dimension 5-Level Questionnaire (EQ-5D-5L). Exploratory end points include pharmacodynamic (PD)/biomarkers, $\mathrm{CR}_{\mathrm{MRD}}$ rate in all patients and the TP53-WT population, OS in the intention-to-treat (ITT) population, secondary efficacy end points in the ITT population, CR during treatment, proportion of allogeneic HSCT regardless of response and leukemia-free survival.

Safety measures include the incidence, severity, nature and seriousness of AEs and the incidence of clinically significant laboratory abnormalities. PK measures include apparent clearance and apparent volume of distribution, maximum concentration, steady-state concentration at the end of a dosing interval, area under the concentrationtime curve (AUC) during one dosing interval, $\mathrm{AUC}_{0-24 \mathrm{~h}}$ and the half-life of idasanutlin, as well as the total 
clearance and volume of distribution of cytarabine and the effect each agent has on the PK of the other. Related to PD/biomarkers, mutational analysis in TP53, FLT3, IDH1, IDH2 and other tumor-associated genes is being conducted and serum macrophage inhibitory cytokine-1 profiles, MDM2 expression, gene signatures and minimal residual disease are being assessed.

\section{Statistical analysis}

A mechanistic simulation model, incorporating cure proportion [26], was used to determine sample size, with a total of 275 OS events in patients with TP53-WT required to provide $82 \%$ power for the primary analysis to detect an OS hazard ratio of 0.67 corresponding to an improvement in median OS from 6 to 9 months in favor of the experimental arm. All patients are randomized to this study regardless of TP53 mutation status. Based on the assumption that $85 \%$ of patients will have TP53-WT disease and 15\% will have TP53-mutant disease, 440 patients are being enrolled in this study.

All randomized patients will be included in the ITT population. Patients will be assessed per the treatment arm to which they were randomized. The TP53-WT population, which will be assessed for the primary end point, includes all randomized patients with TP53-WT as determined by a central laboratory test. OS in the primary end point is defined as the time from randomization to death due to any cause. Kaplan-Meier methodology will be used to estimate survival curves in each treatment arm. The treatment effect of idasanutlin combined with cytarabine versus placebo combined with cytarabine will be described as a hazard ratio using a stratified Cox proportional-hazard regression, including a $95 \%$ CI. To adjust for multiple statistical testing of the primary and key secondary efficacy end points, thus controlling the overall type I error at a two-sided significance level of 5\%, the fixed sequence testing procedure will be utilized. Treatment responses will be assessed at the end of each cycle and summarized by study arm along with the two-sided $95 \%$ CI by the Pearson-Clopper method. Secondary end points such as CR, EFS, overall remission, duration of response, proportion of HSCT following a CR and OS and CR in FLT3, $I D H 1$ and $I D H 2$ populations are part of this analysis. PD/biomarker analyses will be presented using listings and descriptive summary statistics.

All patients who received any amount of idasanutlin, placebo or cytarabine will comprise the all-patient safety population. Patients with TP53-WT who received any amount of study drug will comprise the TP53-WT safety population. All AEs will be assessed using the National Cancer Institute Common Terminology Criteria for Adverse Event version 4.03 grading system.

For PROs, the evaluable patients will include the randomized population with baseline and $\geq 1$ post-baseline PRO assessment(s). PRO evaluable patients will be included in descriptive analyses of visit summaries and change from baseline as well as responder assessments. All PRO analyses will be conducted based on the assigned treatment arm at randomization.

\section{Discussion \& future perspective}

The assessment of futility based on an intermediate end point of confirmed CR corresponded to an integrated Phase II step, which allowed continuous patient enrollment while preserving trial integrity and minimizing bias, since the sponsor and investigators were blinded to the data. Although accelerating development in the AML disease setting was desired at the time of study design, as only later new drugs in targetable subpopulations were approved, skipping Phase II altogether was considered unsound. Integrating a Phase II step with an intermediate end point was therefore considered preferable as risk mitigation to reduce development time, compared with traditional Phase II and III trials, as well as patient exposure, while allowing early stopping for less than expected treatment performance. Absence of an accepted standard of care in R/R AML made the choice of a comparator arm a challenge for this study. While several chemotherapeutic combination therapies are used globally, none has proven superior in clinical trials. Prior example and study design consideration led to selection of a placebo-controlled study design.

The specific design of the MIRROS study with its acceleration of development and reduction of patient exposure made establishment of a clear safety profile a necessity. The benefit of allowing clear assessment of the experimental arm safety profile as well as the possibility to operationalize a randomized blinded study favored selection of a placebo-controlled design. In line with this conclusion, the eligibility criteria limited study enrollment to patients who could also expect benefit from treatment in the control arm.

In mid-2017, MIRROS successfully met the safety and efficacy criteria for continuation without modification, as evaluated during the assessment of the interim futility analysis. As of April 2019, approximately $90 \%$ of the projected patient population was recruited and accrual is still ongoing. An interim efficacy analysis is planned to 
occur when approximately $80 \%$ of the death events have been observed in the TP53-WT population, leading to a potential readout of the data in early 2020. Alternatively, if data are still not mature, the study will proceed to final analysis.

\section{Conclusion}

MIRROS investigates whether idasanutlin in combination with cytarabine may improve OS in patients with $\mathrm{R} / \mathrm{R}$ AML compared with placebo in combination with cytarabine. The results of this study may lead to the availability of a novel treatment option for patients with R/R AML.

\section{Executive summary}

\section{Background}

- For patients with acute myeloid leukemia (AML), the risk of relapse is high and a substantial proportion will have primary refractory disease.

- In relapsed or refractory AML, there is no standard treatment regimen. The prognosis in this population is poor and an unmet medical need remains despite advances in therapeutic approaches.

- Idasanutlin, is a potent small molecule MDM2 inhibitor that disrupts the p53-MDM2 interaction, leading to activation of $\mathrm{p} 53$.

- Idasanutlin in combination with cytarabine demonstrated acceptable safety and promising clinical activity in a Phase I/Ib study in patients with AML. The recommended dose for Phase III evaluation of idasanutlin was also identified in this study.

\section{MIRROS}

- The global, randomized, double-blind, Phase III MIRROS trial is evaluating idasanutlin in combination with cytarabine versus placebo in combination with cytarabine in patients with relapsed or refractory AML.

- MIRROS includes a futility interim analysis that serves as an integrated Phase II step, thus allowing for continuous enrollment of patients and accelerated development.

- The futility interim analysis used a high bar on the odds ratio for durable complete remission and event-free survival and stop/go gating criteria for safety; MIRROS met its continuation criteria in mid-2017.

- Enrollment is ongoing in the Americas, Europe and Asia-Pacific region; $90 \%$ of the projected patient population has been recruited as of April 2019.

- The primary end point is overall survival in the TP53 wild-type population.

- The interim overall survival analysis will allow for treatment benefit to be assessed earlier in this aggressive disease.

\section{Author contributions}

All authors contributed to drafting of the work or revising it critically for important intellectual content as well as read and approved the final version to be published. All authors agree to be accountable for all aspects of the work in ensuring that questions related to the accuracy or integrity of any part of the study are appropriately investigated and resolved. P Fenaux, BM Beckermann, G Martinelli, A Monnet and C Röllig designed the study and provided substantial contributions to its conception. P Montesinos, BM Beckermann, J Esteve, K Gamel, MY Konopleva, G Martinelli, A Monnet, C Papayannidis, A Park, C Récher, R Rodríguez-Veiga, N Vey, A Wei, S-S Yoon and P Fenaux contributed to the acquisition, analysis or interpretation of data for the study. O Catalani and A Monnet have provided statistical support.

\section{Acknowledgments}

We thank the patients and their families, investigators and clinical study sites for their participation. We also thank M Ott for her contributions to this manuscript.

Financial \& competing interests disclosure

This study is sponsored by F. Hoffmann-La Roche, Ltd. P Montesinos was an investigator in Roche clinical trials and received advisory board fees from Roche. J Esteve received advisory board fees and personal fees related to AML therapy from Roche, Novartis, AbbVie, Jazz, Daiichi Sankyo and Astellas. MY Konopleva received advisory board fees from Roche and received grants and personal fees from Roche, Genentech and AbbVie. G Martinelli and C Papayannidis received payment from Roche to their institution for involvement in this study. A Park was previously an employee of Roche and is now an employee of PRA Health Sciences. C Récher received grants and personal fees from Celgene, Sunesis, Amgen, Novartis, Daiichi Sankyo, Jazz and Astellas; personal fees from Incyte, AbbVie, Macrogenics, Otsuka, Roche and Janssen; grants from MaatPharma, Agios and Roche. R Rodríguez-Veiga 
participated in Speakers' Bureau for Teva, Pfizer, Janssen and Novartis; and received travel grants from Sanofi and Daiichi Sankyo. C Röllig received research grants or personal fees from AbbVie, Amgen, Astellas, Bayer, BMS, Celgene, Daiichi Sankyo, Janssen, Jazz, Novartis, Pfizer and Roche. N Vey received personal fees from Roche. A Wei received honoraria from Novartis, Astellas, Pfizer, Macrogenics, AbbVie, Genentech, Servier, Celgene, Amgen, AstraZeneca and Janssen; research funding from Novartis, Celgene, AbbVie, Servier, AstraZeneca and Amgen; is a former employee of the Walter and Eliza Hall Institute; receives a fraction of its royalty stream related to venetoclax; received grants, personal fees and nonfinancial support from Novartis, AbbVie, Servier, Amgen and AstraZeneca; nonfinancial support from Astellas; personal fees and nonfinancial support from Macrogenetics, Genentech, Inc. and Janssen; and receives royalties from AbbVie. S-S Yoon received a research grant from Roche for involvement in this study. P Fenaux received honoraria and research funding from Roche. BM Beckermann, O Catalani, K Gamel and A Monnet are employees of Roche. BM Beckermann holds shares from Roche and Novartis. A Monnet holds shares from Roche. Aaron Park is currently employed at PRA Health Sciences. The authors have no other relevant affiliations or financial involvement with any organization or entity with a financial interest in or financial conflict with the subject matter or materials discussed in the manuscript apart from those disclosed.

Medical writing assistance for this manuscript was provided by P Gadkari and funded by F. Hoffmann-La Roche Ltd.

Ethical conduct of research

All patients provided written informed consent by signing and dating the consent forms prior to study participation. The study protocol, informed consent documents, patient information and other study-related materials were approved by the Institutional Review Board or Ethics Committee at each study site.

Open access

This work is licensed under the Attribution-NonCommercial-NoDerivatives 4.0 Unported License. To view a copy of this license, visit http://creativecommons.org/licenses/by-nc-nd/4.0/

\section{References}

Papers of special note have been highlighted as: $\bullet$ of interest; $\bullet \bullet$ of considerable interest

1. De Kouchkovsky I, Abdul-Hay M. Acute myeloid leukemia: a comprehensive review and 2016 update. Blood Cancer J. 6(7), e441 (2016).

2. Bohl SR, Bullinger L, Rücker FG. New targeted agents in acute myeloid leukemia: new hope on the rise. Int. J. Mol. Sci. 20(8), 1983 (2019).

3. Shallis RM, Wang R, Davidoff A, Ma X, Zeidan AM. Epidemiology of acute myeloid leukemia: recent progress and enduring challenges. Blood Rev. 36, 70-87 (2019).

4. Megías-Vericat JE, Martinez-Cuadron D, Sanz MÁ, Montesinos P. Salvage regimens using conventional chemotherapy agents for relapsed/refractory adult AML patients: a systematic literature review. Ann. Hematol. 97(7), 1115-1153 (2018).

5. Verma D, Kantarjian H, Faderl S et al. Late relapses in acute myeloid leukemia: analysis of characteristics and outcome. Leuk. Lymphoma 51(5), 778-782 (2010).

6. Dohner H, Estey E, Grimwade D et al. Diagnosis and management of AML in adults: 2017 ELN recommendations from an international expert panel. Blood 129(4), 424-447 (2017).

7. Motabi IH, Ghobadi A, Liu J et al. Chemotherapy versus hypomethylating agents for the treatment of relapsed acute myeloid leukemia and myelodysplastic syndrome after allogeneic stem cell transplant. Biol. Blood Transplant. 22(7), 1324-1329 (2016).

8. National Comprehensive Cancer Network (NCCN). Clinical Practice Guidelines in Oncology. Acute Myeloid Leukemia. V2.2020 (2020). www.nccn.org/professionals/physician_gls/pdf/aml.pdf

9. Chène P. Inhibiting the p53-MDM2 interaction: an important target for cancer therapy. Nat. Rev. Cancer 3(2), 102-109 (2003).

10. Moll UM, Petrenko O. The MDM2-p53 interaction. Mol. Cancer Res. 1(14), 1001-1008 (2003).

11. Kubbutat MH, Jones SN, Vousden KH. Regulation of p53 stability by Mdm2. Nature 387(6630), 299-303 (1997).

12. Haupt Y, Maya R, Kazaz A, Oren M. Mdm2 promotes the rapid degradation of p53. Nature 387(6630), 296-299 (1997).

13. Momand J, Jung D, Wilczynski S, Niland J. Nucleic Acids Res. 26, 3453-3459 (1998).

14. Melo MB, Ahmad NN, Lima CS et al. Mutations in the $p 53$ gene in acute myeloid leukemia patients correlate with poor prognosis. Hematology 7(1), 13-19 (2002).

15. Kadia TM, Jain P, Ravandi F et al. TP53 mutations in newly diagnosed acute myeloid leukemia: clinicomolecular characteristics, response to therapy and outcomes. Cancer 122(22), 3484-3491 (2016).

16. Martinelli G, Pappayannidis C, Yee K et al. Phase 1B results of idasanutlin + cytarabine (Ara-C) in acute myelogenous leukemia (AML) patients (pts). Haematologica 101(S1), Abstract S504 (2016). 
17. Ding Q, Zhang Z, Liu JJ et al. Discovery of RG7388, a potent and selective p53-MDM2 inhibitor in clinical development. J. Med. Chem. 56(14), 5979-5983 (2013).

- Describes the identification and characterization of MDM2 inhibitor idasanutlin.

18. Higgins B, Tovar T, Glenn K et al. Antitumor activity of the MDM2 antagonist RG7388. Mol. Cancer Ther. 12(Suppl. 11), Abstract B55 (2013).

- Demonstrates the antitumor activity of idasanutlin as monotherapy and in combination with cytarabine in vivo.

19. Yee K, Martinelli G, Vey N et al. Phase I/Ib study of RG7388, a potent MDM2 antagonist, in acute myelogenous leukemia (AML) patients (pts). Blood 124(21), Abstract 116 (2014).

20. Kotler E, Shani O, Goldfeld G et al. A systematic p53 mutation library links differential functional impact to cancer mutation pattern and evolutionary conservation. Mol. Cell. 71(1), 178-190 (2018).

21. Kato S, Han SY, Liu W et al. Understanding the function-structure and function-mutation relationships of $\mathrm{p} 53$ tumor suppressor protein by high-resolution missense mutation analysis. Proc. Natl. Acad. Sci. USA 100(14), 8424 (2003).

22. Faderl S, Gandhi V, O'Brien S et al. Results of a Phase I-II study of clofarabine in combination with cytarabine (ara-C) in relapsed and refractory acute leukemias. Blood 105(3), 940-947 (2005).

23. Archimbaud $\mathrm{E}$, Leblond $\mathrm{V}$, Michallet $\mathrm{M}$ et al. Intensive sequential chemotherapy with mitoxantrone and continuous infusion etoposide and cytarabine for previously treated acute myelogenous leukemia. Blood 77(9), 1894-1900 (1991).

24. Dohner H, Estey EH, Amadori S et al. Diagnosis and management of acute myeloid leukemia in adults: recommendations from an international expert panel, on behalf of the European LeukemiaNet. Blood 115(3), 453-474 (2010).

25. Hunsberger S, Zhao Y, Simon R. A comparison of Phase II study strategies. Clin. Cancer Res. 15(19), 5950-5955 (2009).

- Provides rationale for clinical trial designs that integrate Phase II and Phase III features.

26. Rufibach K, Heinzmann D, Monnet A. Integrating Phase II into Phase III based on an intermediate endpoint while accounting for a cure proportion-with an application to the design of a clinical trial in acute myeloid leukemia. Pharm. Stat. 19(1), 44-58 (2019).

-. Provides rationale for and details the statistical design of the futility interim analysis in MIRROS. The futility interim analysis integrates Phase II criteria into a Phase III study, mitigating the risk of moving from Phase I to Phase III development.

27. Medeiros BC. Interpretation of clinical endpoints in trials of acute myeloid leukemia. Leuk. Res. 68, 32-39 (2018).

28. Nemunaitis J, Young A, Ejadi S et al. Effects of posaconazole (a strong CYP3A4 inhibitor), two new tablet formulations and food on the pharmacokinetics of idasanutlin, an MDM2 antagonist, in patients with advanced solid tumors. Cancer Chemother. Pharmacol. 81(3), 529-537 (2018).

29. Ramos NR, Mo CC, Karp JE, Hourigan CS. Current approaches in the treatment of relapsed and refractory acute myeloid leukemia. J. Clin. Med. 4(4), 665-695 (2015).

30. Yee K, Martinelli G, Assouline S et al. Phase Ib study of the MDM2 antagonist RG7112 in combination with 2 doses/schedules of cytobarine. Blood 122(21), Abstract 498 (2013).

31. Lowenberg B, Pabst T, Vellenga E et al. Cytarabine dose for acute myeloid leukemia. N. Engl. J. Med. 364(11), 1027-1036 (2011).

32. Lowenberg B. Sense and nonsense of high-dose cytarabine for acute myeloid leukemia. Blood 121(1), 26-28 (2013).

33. Roboz GJ, Rosenblat T, Arellano M et al. International randomized Phase III study of elacytarabine versus investigator choice in patients with relapsed/refractory acute myeloid leukemia. J. Clin. Oncol. 32(18), 1919-1926 (2014).

34. Arber DA, Orazi A, Hasserjian R et al. The 2016 revision to the World Health Organization classification of myeloid neoplasms and acute leukemia. Blood 127(20), 2391-2405 (2016).

35. Baugh EH, Ke H, Levine AJ, Bonneau RA, Chan CS. Why are there hotspot mutations in the TP53 gene in human cancers? Cell Death Differ. 25(1), 154-160 (2018). 
